\title{
Investigando el concepto de nativos digitales en la Universidad
}

\section{Investigating the concept of digital natives in the University}

\author{
Eduardo Escalante Gómez (Chile) \\ escalante.gomez@gmail.com \\ Universidad Juan Agustín Maza \\ Máster en Ciencias Sociales
}

\section{Resumen}

En este estudio se explora cómo se involucran con la tecnología los alumnos de primer año de universidad en su vida académica y en su vida cotidiana; y hasta qué punto se verifica la noción de "nativos digitales" y se explora en qué medida los usos y preferencias por tecnologías particulares se relacionan con las identidades que ellos adoptan en los contextos académicos y de actuación cotidiana. Se emplea un enfoque cuantitativo (survey) y cualitativo (entrevista semi estructuradas). Los hallazgos muestran que estos

\begin{abstract}
Summary
AThis study explores how the students enrolled in the first year of university engage with technology in their academic and daily lives; and to what extent it verifies the notion of "digital natives" and explores the uses and preferences for particular technologies related to the identities that they adopt in academic settings and daily activities. It employs a quantitative approach (survey) and qualitative (semi- structured interview).

The findings show that these students have different patterns of technological
\end{abstract}

PARA CItAR ESte artículo / to CIte thIS ARTICle Escalante Gómez, E. (2015). Investigando el concepto de nativos digitales en la Universidad, Poliantea, $12(22)$. 
alumnos tienen diferentes patrones de involucramiento tecnológico en diferentes contextos, y que las tecnologías y sus capacidades no siempre son transferibles entre contextos.

En cuanto a limitaciones del estudio, se puede señalar que la muestra se tomó de una sola institución. También es necesario indicar que hay una falta de lenguaje compartido sobre la definición de este tipo de tecnologías, lo que de algún modo limitó a los participantes en el estudio para articular su compromiso con las actividades basadas en la tecnología. El estudio es un aporte empírico a la discusión de algunas generalizaciones que están instaladas en muchas instituciones, a modo de agregar antecedentes para su problematización y examinar las maneras como se están gestionando las nuevas tecnologías en relación con los procesos de enseñanza y aprendizaje.

Palabras clave: nativos digitales, estudiantes universitarios, nuevas tecnologías, educación. involvement in different contexts and that the technologies and their capabilities are not always transferable between contexts. In regards to the limitations of the study, it should be noted that the sample was taken from a single institution. It is also necessary to indicate that there is a lack of shared language on the definition of this type of technologies which in some way limited the participants in the study to articulate their commitment to activities based on technology.

The study is an empirical contribution to the discussion of some generalizations made in many institutions, in order to add background to their problematic and examine the ways new technologies are managed in relation to the processes of teaching and learning.

Key Words: Digital Natives, university students, new technologies, education. 


\section{Introducción}

Se observa un incremento en la incorporación de las nuevas tecnologías en las universidades para apoyar los procesos de enseñanza y aprendizaje. Se postula de uno u otro modo que los alumnos que ingresan a la universidad traen experiencias tecnológicas obtenidas en distintos contextos. En algunos casos se argumenta que estaríamos enfrentados a una cuestión generacional, apoyándose en algunas elaboraciones teóricas derivadas de evidencias anecdóticas. Se trata de quienes postulan el concepto de "nativos digitales" o la "generación de la Red", asumiendo que los más jóvenes tendrían la habilidad natural y las motivaciones para usar las tecnologías en todos los aspectos de sus vidas (Prensky, 2001; Tapscott, 1998).

No obstante, nuevas evidencias han cuestionado la naturaleza homogénea de este grupo, porque se ha hallado una diversidad de patrones de acceso y uso de los jóvenes de la tecnología (Oliver \& Goerke, 2007). Esto ha hecho que los investigadores sean precavidos respecto de esa retórica y los supuestos que describirían a esa generación. Este estudio investiga cómo se involucran con la tecnología los alumnos de primer año de universidad en su vida académica y en su vida cotidiana. Se examina hasta qué punto se verifica la noción de "nativos digitales" y cómo los alumnos seleccionan y adaptan las tecnologías para apoyar sus metas y estrategias de aprendizaje. Además, se explora en qué medida los usos y preferencias por tecnologías particulares se relacionan con las identidades que ellos adoptan en los contextos académicos y de actuación cotidiana. 


\section{Marco teórico}

Hay una variedad de nombres para describir a los alumnos que estudian en la universidad. Entre ellos, nativos digitales, la generación Google o la generación milenio. Todos estos nombres se han usado para destacar el significado y la importancia de las nuevas tecnologías en la vida de los jóvenes (Gibbons, 2007).

Para algunos, las nuevas tecnologías constituyen un rasgo sustantivo en la vida de las nuevas generaciones que permitirían predecir un cambio fundamental en la manera como los jóvenes se comunican, socializan, crean y aprenden (Prensky, 2001; Gibbons, 2007). Típicamente, quienes apoyan este concepto ven las diferencias entre aquellos que son y los que no son nativos digitales, en función de su fecha de nacimiento.

La noción de la generación de "nativos digitales", según la proponen Prensky (2001) y Tapscott (1998), descansa fundamentalmente en la similaridad de las actitudes de los jóvenes hacia la tecnología y su uso. No obstante, diferentes estudios han probado que lo que se observa es un involucramiento muy diverso en su naturaleza (Margaryan, Littlejohn y Vojt, 2011); esto se confirmaría en esta investigación. Esta diversidad sería el resultado de diferentes motivaciones, influencias y contextos de los alumnos que interactúan con la tecnología, en este caso, alumnos de primer año de universidad.

La revisión de la literatura revela que varios de los supuestos subyacentes en la noción de nativos digitales se han visto cuestionados recientemente.

Una excelente y exhaustiva revisión sobre mitos y realidades acerca de los nativos digitales es el artículo de Cabra Torres y Marciales Vivas (2009). En este escrito, quedan en evidencia los vacíos conceptuales y metodológicos, así como las diversas maneras de investigar el concepto 'nativos digitales'. Como señalan las autoras, uno de los aspectos preocupantes son las barreras generacionales y culturales que se han tratado de establecer a partir del concepto.

La literatura sobre los nativos digitales atribuye la alta alfabetización técnica de los jóvenes a su exposición continua con la tecnología (Prensky, 2001; Tapscott, 1998). No obstante, algunos estudios han comprobado que el nivel de acceso de los jóvenes a la tecnología está influenciado por el estatus socioeconómico 
(Hargittai, 2010) y por el contexto del país (Thinyane, 2010). Por otro lado, se ha asumido que los jóvenes se mantienen conectados a las últimas tendencias de la tecnología. No obstante, se ha verificado que se trata de una minoría que tiene acceso, en términos de recursos, a las novedades tecnológicas. También los estudios han hallado que los jóvenes exploran la disponibilidad y nuevas capacidades de las tecnologías cuando tienen necesidades específicas; además, se observa un conocimiento limitado de los diversos avances tecnológicos (Margaryan et al. 2011).

Algunos autores sostienen que los nativos digitales se involucran comúnmente en la creación de contenidos que comparten en línea (Lorenzo et al., 2007). Sin embargo, hay pocos ejemplos de creación de contenidos (Ferri y Pozzali, 2012). Se ha observado un bajo nivel de adopción, especialmente cuando se trata de las últimas innovaciones tecnológicas (blogs, wikis, RSS feeds). Hipotéticamente se piensa que se debería a la falta de utilidad de estas para la vida personal de los alumnos. La mayor frecuencia observada se da en relación con el uso de herramientas de comunicación.
Todo lo anterior sugiere que los jóvenes no usan una amplia gama de tecnologías, como fue sugerido en alguna literatura sobre los nativos digitales (Prensky, 2001; Tapscott, 1998).

En síntesis, no se trata de un concepto sobre el que exista consenso. En este estudio se ha tratado de abordar su investigación usando un encuadre teórico que combina los conceptos de la apropiación de la tecnología con la teoría de la identidad, en orden a abordar el diseño, análisis de datos e interpretación de esta investigación. Es una manera de aportar a los investigadores e instituciones con datos empíricos para la reflexión sobre lo que ocurre en diferentes contextos y realidades. No se trata de un fenómeno simple. El concepto de nativos digitales ha sido novedoso, entusiasmando a muchos que tratan de entender el impacto de la tecnología en el aprendizaje y la enseñanza.

De a poco ha ido quedando claro que lo que ocurre en la vida cotidiana con la tecnología no significa una transferencia lineal al ámbito académico, y el uso de la tecnología podría significar el dominio de un instrumento, pero no necesariamente el dominio de los procesos intelectuales que demanda la 
formación cultural y científica a través de procesos de enseñanza y aprendizaje; quizás más profundo aún, el proceso lúdico de uso de la tecnología puede actuar como un distorsionador de los aprendizajes e incluso obstaculizarlo.

La literatura revela que las investigaciones sobre cómo las personas adoptan y aceptan la tecnología en sus vidas ha conducido a desarrollar diferentes modelos teóricos que han puesto en evidencia que el proceso de adopción significa que los adoptantes individuales pasan desde la concienciación a la aceptación completa, en nuestro caso, de una innovación. Las decisiones de adopción de innovaciones se diferencian de otros tipos de toma de decisiones por la novedad relativa de estas innovaciones y la mayor incertidumbre asociada a este tipo de decisión.

Estos modelos han ido examinando las motivaciones y comportamiento de quienes adoptan la tecnología en términos de percepción de uso, percepción de utilidad y actitud hacia la tecnología. Este tipo de enfoque ha pasado a formar parte de la investigación sobre la adopción de la tecnología (Straub, 2009).

Por otro lado, se ha puesto de manifiesto que la adopción de la tecnología implica diferentes procesos: cognitivo, afectivo (evaluativo) y de comportamiento (conativa). Y en función de estos procesos, la persona actúa de una determinada manera.

Basados en el constructivismo social y los estudios de los jóvenes que usan los smartphones, Carroll et al. (2001), desarrollaron un modelo de apropiación de la tecnología en cuanto diseño y en cuanto a uso. Desde el punto de vista de este modelo, la tecnología puede significar diferentes cosas para diferentes personas como resultado de los procesos de negociación e interpretación en sus contextos específicos (Hine, 2000). En otras palabras, la tecnología puede dar forma y es formada por la sociedad (Carroll et. al., 2001, 2002).

En esta investigación, se define la apropiación de la tecnología en términos de las maneras que las personas evalúan y adoptan, adaptan e integran la tecnología en sus comportamientos cotidianos. En esta interpretación se cruzan los deseos de las personas, las capacidades de la tecnología, y el impacto de la sociedad. Sí es lícito señalar que no se postula una ruta determinística para explicar lo que es la tecnología por la manera como se usa; la 
tecnología se puede usar de diferentes maneras e interpretarse de diferentes maneras por las personas. Una misma tecnología puede ser adaptada de diferentes maneras y en diferentes contextos.

Por otra parte, en este estudio se incorporó la teoría de la identidad a partir de las elaboraciones de Stryker $(1980,1997)$, quien provee una perspectiva útil para considerar cómo los alumnos de primer año de universidad se apropian de la tecnología en los diferentes contextos de sus vidas. A partir de lo que postula esta teoría, se podría señalar que los alumnos pueden tener múltiples identidades como resultado de sus múltiples roles desempeñados en la sociedad. Por otro lado, el involucramiento con la tecnología podría verse afectado por las identidades múltiples de los alumnos, tanto en entornos en línea como fuera de línea.

La identidad también ha sido usada al considerar la validez de constructos generacionales como el de "nativos digitales", o la "Generación de la Red" (Palfrey y Gasser, 2008). Desde este punto de vista, resultaría útil para interpretar cómo los individuos enfocan la apropiación tecnológica y cómo ellos son afectados por la tecnología.
En síntesis, este estudio se apoya en estos enfoques teóricos para examinar la adopción, adaptación y transferencia de la tecnología entre los contextos cotidianos y académicos de los alumnos. Además, se complementó mediante el análisis de cómo los roles de un individuo en la sociedad impactan la adopción y uso de la tecnología.

\section{Materiales y métodos}

Se diseñó un enfoque que empleó un survey y entrevistas semi estructuradas, en orden a lograr examinar en profundidad la diversidad y complejidad de las actividades de los alumnos de primer año de universidad que están basadas en la tecnología.

La primera fase consistió en la administración de un survey aplicado a los alumnos sobre el acceso y uso de la tecnología. Se utilizó una muestra de 150 alumnos. Se intentó identificar tendencias y patrones de involucramiento de los alumnos con las tecnologías.

El survey recopiló datos demográficos incluyendo, edad, género, carrera que se estudia. Se reunieron también datos administrativos que permitieron señalar que se trata 
de una cohorte nacida en los años 1997, 1998. También se les pidió a los alumnos que autoevaluaran su habilidad para usar la tecnología, ya sea nivel principiante, intermedio, o avanzado. Además, se incluyeron preguntas para identificar el tipo de tecnología al que tenían acceso, bien sea como propietarios, de acceso compartido o ningún tipo de acceso. El listado de tecnologías que se incluyó se refiere al contexto de la vida cotidiana o de la vida académica, incluyendo el uso de PCs, laptops, tabletas, cámaras digitales, acceso a internet. También se hicieron preguntas sobre el uso de las tecnologías en el contexto cotidiano y en el contexto académico.

Los datos fueron recopilados durante el año 2014. De los 150 participantes, un $85,3 \%$ nacieron entre 1997 y 1998. La distribución en términos del género estuvo hacia las mujeres con $64 \%$ de mujeres y un $35,7 \%$ de hombres.

En la segunda fase, se realizaron entrevistas semi estructuradas, para lo que se tomaron 14 casos seleccionados a partir de la diversidad detectada en el survey, tratando de que fueran representativos de los aspectos claves de este estudio. Los casos seleccionados incluyeron el rango de tecnologías de acceso, género, edad, y carrera de estudio. Se trataron de recopilar sus niveles de adopción y uso de la tecnología como también sus estrategias y metas de aprendizaje. También se usó para explorar en qué medida los escenarios de enseñanza generan requerimientos de uso de la tecnología digital o si ellos individualmente buscan apoyarse en las nuevas herramientas.

$\mathrm{El}$ análisis de los datos se realizó creando una matriz de codificación que identificó los indicadores y expresiones asociadas tanto a la apropiación de la tecnología como a la identidad. Para este efecto, el estudio se apoyó en el enfoque de Miles y Huberman (1994).

\section{Resultados}

Primero se analizaron los resultados del survey y posteriormente se exploraron los datos obtenidos en las entrevistas. Se trató de acotar el análisis a las preguntas y objetivos de la investigación sobre relación con el involucramiento de los alumnos con la tecnología para obtener una aproximación sobre el uso y adopción de la tecnología en sus vidas cotidiana y académica, y los procesos relacionados con la identidad. 
En la exposición que sigue se van combinando los resultados del survey y de las entrevistas.

Los alumnos de las carreras de Humanidades y Ciencias Sociales representaron un 56\%, y los de las carreras de Ciencia, un $44 \%$. Solamente un 23,2\% de los alumnos clasificaron su habilidad con la tecnología como avanzada; la mayoría (67\%), se catalogaron ellos mismos como nivel intermedio, y un $8,5 \%$ se clasificaron como principiantes. En términos de género, un $34 \%$ de los varones se clasificaron como usuarios avanzados de la tecnología y las mujeres un $17,4 \%$.

Respecto al acceso a la tecnología, un $92,4 \%$ señalaron tener acceso a PC, un $84,3 \%$, a laptops; un $60 \%$, a smartphones; un $96,6 \%$, a pen drives; un $88,9 \%$ a cámaras digitales, y un $35 \%$, a tabletas. Un $21,2 \%$ indicaron tener acceso limitado o no acceso a una computadora.

Un $75,8 \%$ indicaron tener acceso a la información en línea, un 98,7\% indicaron tener acceso al mail, y un $85 \%$ acceso a la creación de presentaciones. Respecto de la participación en la redes sociales, se halló una proporción superior de alumnos en las carreras de Humanidades y Ciencias Sociales, un 70,5\%, mientras que en las carreras de Ciencia, su participación es de 52,7\%.

El número de los alumnos, respecto del uso de tecnologías que apoyan las actividades académicas, es muy bajo en relación con el uso que ellos hacen en el día a día. Los patrones de uso son consistentes con aquellos observados en otros estudios (García y Qin, 2007).

A través del estudio, se pudo ir constatando claramente que no todos los alumnos de primer año de universidad calzan en un estereotipo generacional simple. Hay investigadores que han tratado de formular clasificaciones alternativas de los jóvenes respecto de sus hábitos tecnológicos (Ferri et al., 2008; Morgan y Bullen, 2011). Este estudio confirmaría que la complejidad del uso de la tecnología que realizan los jóvenes hace problemática la elaboración de clasificaciones. Esta complejidad fue detectada en este estudio, dada la diversidad de uso que hacen los alumnos de las distintas tecnologías.

Los datos del survey mostraron que, en todos los casos, los alumnos usan el email como forma de comunicación básica; en las entrevistas se descubrió que algunos lo usan para la transferencia de archivos entre 
dispositivos. Algunos alumnos indicaron que usan las herramientas de conexión social para comunicarse con los amigos, otros para los eventos sociales cada a cara. Esta variedad de uso de la tecnología se combina con los diferentes contextos y factores motivacionales, lo que crea una complejidad que hace problemática la identificación de grupos consistentes basada en las características tecnológicas. Esto indicaría que enfocar la investigación de esta manera no sería particularmente útil para construir una mayor comprensión del uso que hacen de la tecnología los alumnos en relación con la enseñanza y el aprendizaje.

El estudio también abordó el cómo los alumnos incorporan la tecnología en sus prácticas de estudio. La noción de nativos digitales parte de determinados supuestos sobre las actitudes de los jóvenes hacia la educación y el rol de la tecnología en el aprendizaje, que estarían en contradicción con las actuales prácticas de enseñanza y aprendizaje en la universidad. Por ejemplo, se ha sugerido que los métodos tradicionales de enseñanza no serían capaces de involucrar a los alumnos cuyas actitudes hacia la educación serían totalmente diferentes.
(Prensky, 2001; Tapscott, 1998). Se ha señalado que cambios en las actitudes de los alumnos, comportamiento y preferencias resultantes de su exposición a la tecnología a través de sus vidas, estaría creando una manera fundamental diferente de actuación (Prensky, 2001). Este tipo de alumno estaría demandando el uso de más tecnología, requiriendo un mayor rol de la misma (Dede, 2005; Prensky, 2001).

En esta investigación se halló que el enfoque de los alumnos hacia el estudio académico es relativamente tradicional en términos de sus técnicas de estudio, así como la preparación para las evaluaciones y exámenes. En las entrevistas, los alumnos informaron actividades tales como revisar las notas de clases, realizar pruebas en línea, escuchar las grabaciones de las clases constituían parte de su estudio académico. Ellos usan la tecnología para producir documentos y presentaciones, para comunicarse con los docentes y compañeros, y acceder a los recursos de aprendizaje que están en línea. A través del estudio se encontraron pocos ejemplos de uso de la tecnología de manera innovadora en términos de apoyo a sus procesos de aprendizaje académico. No 
se observó incompatibilidad con los métodos tradicionales de enseñanza y aprendizaje.

Estudios similares observaron que los alumnos con altos niveles de alfabetización tecnológica se sentían cómodos con los métodos de enseñanza y aprendizaje en uso (García y Qin, 2007). No se detectó una insatisfacción con los actuales métodos vigentes en la universidad. Esto desafía de manera sustantiva la postura de Prensky, 2001 y Tapscott, 1998.

Las diferencias entre el tipo de carrera que cursan también tendría influencia en los tipos de enseñanza y aprendizaje, según la valoración que hacen los alumnos. Por ejemplo, a los estudiantes de las carreras de Ciencia les gustaba disponer de material en línea y revisar contenidos, mientras que a los de Humanidades y Ciencias Sociales, les gustaba más encontrar sus propios recursos de aprendizaje y en especial hacer uso de los videos de Youtube. Este tipo de diferencias también han sido detectadas en otros estudios (Selwyn, 2008).

Los hallazgos del estudio también sugieren que el interés de los alumnos por ciertas tecnologías en su vida diaria no se traducen necesariamente en un deseo o habilidad para adaptarlas al contexto académico. Algunos señalaron, por ejemplo, que herramientas de las redes sociales como Facebook no eran útiles para sus estudios académicos. Hay una variedad de argumentaciones de los alumnos que revelan que no necesariamente se transfiere el uso de las tecnologías al contexto académico. Esto estaría revelando que los alumnos requieren apoyo si se trata de usar la tecnología para los procesos de enseñanza y aprendizaje, lo que es confirmado por diferentes autores (por ejemplo, Gros et al., 2012).

En resumen, este estudio muestra que cuando se selecciona y adopta la tecnología para apoyar la enseñanza y el aprendizaje, se requiere orientar a los alumnos. Estos hacen un uso frecuente de la tecnología, pero no transfieren su uso a la vida académica. Hay poca evidencia de que los alumnos de primer año de universidad usen la tecnología de manera innovadora, para efectos de procesos de aprendizaje académico. Tampoco se hallaron evidencias que indiquen que existe un deseo por un cambio radical del enfoque educativo, al contrario de lo formulado por la literatura sobre la generación de nativos digitales (Palfrey y Gasser, 2008; Prensky, 2001; Tapscott, 1998). 
También se exploró la identidad que los alumnos de primer año de universidad adoptan en sus vidas cotidianas y en el estudio académico, y el impacto de estas en el uso y preferencia por las tecnologías. Como se ha señalado más arriba, la teoría de la identidad propone que las personas tienen múltiples identidades que se definen por los diferentes roles que desempeñan en la sociedad (Stryker y Burke, 2000). Existen estudios previos sobre el rol de la tecnología en la identidad de los jóvenes (Turkle, 1984).

Cada alumno entrevistado señaló que usaba la tecnología a partir de diferentes propósitos, su nivel de habilidad técnica, el acceso disponible, pero que también influía el tipo de uso y acceso de sus pares. Esto es consistente con evidencias que indican, por ejemplo, que el acceso de los smartphones correspondería más a un estilo de vida que a una perspectiva tecnológica; quieren agregar valor a sus estilos de vida, satisfacer necesidades sociales y lúdicas que refuercen su identidad como grupo. Esta variedad de desafíos que enfrentan los alumnos, desafían el supuesto de la naturaleza homogénea de la generación denominada nativos digitales. Los hallazgos obtenidos indicarían que, si bien algunos alumnos muestran características que los incluirían en la denominada generación de nativos digitales, estas no son aplicables para todos, y se requiere un mayor análisis para identificar la diversidad. Debido a esta pluralidad hay pocos patrones de uso de la tecnología que puedan ser identificados. Sin embargo, hay estudios que los han identificado (Dahlstrom, 2012).

La mayoría de los alumnos en el estudio demostraron una fuerte conexión con su identidad como estudiantes. Esto se hizo evidente por sus estrategias y enfoques para enfrentar los estudios. Pero sus actitudes hacia la tecnología variaron de manera considerable, independientemente de sus fuertes identidades como alumnos: a modo de ejemplo, tres de ellos manifestaron poca adhesión a la tecnología. Sí es preciso señalar que dada la complejidad del término identidad, este puede ser definido de distintas maneras, lo cual fue un desafío de la investigación y se trató de buscar la forma que permitiera a los entrevistados hablar de su identidad. Se observaron dificultades para articular cómo ellos perciben sus roles sociales, y el papel de la tecnología en relación 
a estos roles. Igualmente se les preguntó sobre sus hobbies e intereses.

\section{Discusión}

Los hallazgos de este estudio muestran que estos alumnos tienen diferentes patrones de involucramiento tecnológico en diferentes contextos, y que las tecnologías y sus capacidades no siempre son transferibles entre contextos. Además, la introducción de las tecnologías para apoyar la enseñanza y el aprendizaje requieren que se los familiarice con ellas y se les ayude a usarlas en el contexto académico, dado que no existiría una transferencia automática de las capacidades tecnológicas.

Se empleó un encuadre teórico que permitió mirar el fenómeno en cuestión desde nuevas perspectivas. Se usó la teoría de la apropiación tecnológica y la teoría de la identidad para examinar cómo los alumnos de primer año de universidad eligen y adaptan las tecnologías a partir de sus necesidades en los diferentes contextos de sus vidas. El uso de la apropiación de la tecnología fue útil para considerar las influencias y motivaciones que impactan la selección que hacen los estudiantes para diferentes propósitos en su vida cotidiana y como parte de sus estudios académicos.

Se logró identificar una diversidad respecto del uso y acceso a la tecnología, pero no se detectó un rango muy amplio, como sugieren algunos autores (Dede, 2005; Prensky, 2001; Tapscott, 1998), y se halló que la tecnología que se usa en un contexto no es necesariamente transferible a otro. Esto último es consistente con otros estudios (Margaryan et al., 2011). En adición, el estudio mostró, de manera contraria a los supuestos del concepto de nativos digitales (Prensky, 2001), que los alumnos de primer año de universidad no mostraron enfoques radicalmente diferentes respecto de los enfoques y preferencias hacia el aprendizaje, lo que indica que no es útil apoyarse en la noción de una generación homogénea de nativos digitales al discutir la integración de la tecnología en la enseñanza y el aprendizaje en la universidad. Esto es de vital importancia al considerar las inversiones en tecnologías como en los diseños pedagógicos.

En relación, específicamente, con la práctica de la enseñanza y el aprendizaje, los hallazgos de este estudio sugieren que las actividades de aprendizaje deberían diseñarse de 
tal modo que cubran la variedad de alumnos con diversos niveles en términos de capacidades tecnológicas, dando el necesario apoyo a aquellos con bajos niveles de competencia en esta área (Gros et al., 2012).

En síntesis, respecto de la pregunta investigativa que buscaba determinar hasta qué punto el involucramiento con la tecnología de los alumnos de primer año calzaba con el supuesto de la generación de nativos digitales, indicó que existen múltiples variaciones que hacen que este concepto sea limitado. Esto sugiere que basarse en cuestiones generacionales parecería incorrecto, por lo que se requiere explorar en mayor profundidad la diversidad y complejidad en el uso de la tecnología. Por otro lado, al examinar la relación entre las tecnologías que usan y prefieren los alumnos y las identidades que adoptan en sus vidas cotidiana y académica, los hallazgos revelan que los alumnos usan ciertas tecnologías en los diferentes contextos de sus vidas. Su identidad académica parece no tener un impacto en la frecuencia o rango de uso de la tecnología, pero se observa que los alumnos con una fuerte identidad académica hacen un uso de las tecnologías provistas por la universidad. Sin embargo, hay que señalar que no se pudo explorar a cabalidad la relación entre la identidad y el involucramiento con la tecnología.

Finalmente, el examen de la noción de nativos digitales resulta importante por el uso público de este concepto y por su impacto en la gestión de la tecnología en las universidades. Su uso inapropiado puede afectar negativamente las interacciones docentes-alumnos e introducir una generalización con carácter determinista. Hay que considerar que Prensky (2009), ha modificado su posición. En la actualidad acepta que personas mayores puedan ser nativos digitales, pero en muchos entornos universitarios se sigue perpetuando la creencia de la división entre dos generaciones: jóvenes y adultos.

En cuanto a limitaciones del estudio, se puede señalar que la muestra se tomó de una sola institución. La muestra para el estudio cualitativo mediante entrevistas empleó el enfoque del muestreo con propósito (Creswell, 1998), en orden a incluir una variedad de casos que podrían ser comparados entre ellos y con el perfil típico de un nativo digital. El número de casos fue limitado, pero se empleó el número mínimo recomendado para este tipo de estudio (Creswell, 2002). 
También es necesario indicar que hay una falta de lenguaje compartido sobre la definición de este tipo de tecnologías, lo que de algún modo limitó a los participantes en el estudio para articular su compromiso con las actividades basadas en la tecnología. Del mismo modo, los participantes tuvieron dificultades para articular cómo sus identidades se relacionan con el uso de la tecnología.

\section{Referencias}

Cabra Torres, F.; Marciales Vivas, G. (2009). Mitos, realidades y preguntas de investigación sobre los 'nativos digitales': una revisión. Universitas Psychologica, 8(2), 323-338.

Carroll, J.; Howard, S.; Vetere, F.; Peck, J. y Murphy, J. (2001). Identity, power and fragmentation in cyberspace: technology appropriation by young people. In G. Finnie, D. Cecez-Kecmanovic, \& B. Lo (Eds.), Proceedings of the $12^{\text {th }}$ Australasian Conference on Information Systems (ACIS). 1, 95-102.

Carroll, J.; Howard, S.; Vetere, F.; Peck, J. y Murphy, J. (2002). Just want do the youth of today want?
Technology appropriation by young people, In R. H. Spraque (Ed.), Proceedings of the $35^{\text {th }}$ Hawaii International Conference on system Sciences (HICSS-35), Maui, Hawaii.

Creswell, J. W. (2002). Educational research: Planning, conducting, and evaluating quantitative and qualitative research. Upper Saddle River, NJ: Merrill/Pearson Education, Inc.

Creswell, J. W. (1998). Qualitative Inquiry and Research Design: Choosing Among Five Traditions, Thousand Oaks, CA: Sage Publications.

Dahlstrom, E. (2012). ECAR Study of Undergraduate Students and Information Technology, 2012 (Research Report). Louisville, CO: EDUCAUSE Center for Applied Research.

Dede, C. (2005). Planning for Neomillennial Learning Styles. EDUCAUSE Quarterly, 28(1).

García, P. y Qin, J. (2007). Identifying the generation gap in higher education, Innovate, 3(4).

Ferri, P. y Pozzali, A. (2012). University Students and Social Media: Reflections from an Empirical Research. Journal of Universal Computer Science, 18(3), 377-392. 
Gibbons, S. (2007). Redefining the roles of information professionals in higher education to engage the net generation, Keynote Paper presented at EDUCAUSE Australasia 2007.

Gros, B.; García, I. y Escofet, A. (2012). Beyond the Net Generation Debate: A Comparison of Digital Learners in Face-to-Face and Virtual Universities. The International Review of Research in Open and Distance Learning, 13(4), 190-210.

Hargittai, E. y Hinnant, A. (2008). "Digital Inequality. Differences in Young Adults' Use of the Internet". Communication Research, 35(5), 602-621.

Hargittai, E. (2010). Digital na(t) ives? Variation in Internet skills and uses among members of the "Net Generation". Sociological Inquiry, 80(1), 92-113.

Hine, Christine (2000). Etnografía Virtual. Barcelona: Editorial UOC. Colección Nuevas Tecnologías y Sociedad. 2004.

Kennedy, G.; Judd, T.S.; Churchward, A.; Gray, K. y Krause, K.-L. (2008). First year students' experiences with technology: Are they really digital natives? Australasian
Journal of Educational Technology, 24(1), 108-122.

Lorenzo, G., Oblinger, D. y Dziuban, C. (2007) How choice, cocreation, and cultures are changing what it means to be net savvy. Educause Quarteley, 30(1).

Margaryan, M.; Littlejohn, A. y Vojt, G. (2011) Are digital natives a myth or reality? Students' use of technologies for learning. Computers \& Education, 56(2), 429-440.

Miles, M. B., y Huberman, A. M. (1994). Qualitative data analysis: An expanded sourcebook. Thousand Oaks, CA: Sage.

Morgan, T., y Bullen, M. (2011). Digital learners in higher education: A research project update. Journal of Distance Education, 25(1).

Oliver, B. y Goerke, V. (2007). Australian undergraduates' use and ownership of emerging technologies: Implications and opportunities for creating engaging learning experiences for the Net Generation. Australasian Journal of Educational Technology, 23(2), 171-186.

Palfrey, J. y Gasser., U. (2008). Born digital: Understanding the first 
generation of digital natives. New York: Basic Books.

Prensky, M. (2001). Digital Natives, Digital Immigrants. On the Horizon, 9(5).

Prensky, M. (2009). H. sapiens digital: from digital immigrants and digital natives to digital wisdom. Innovate Journal of Online Education, 5.

Selwyn, N. (2008). An investigation of differences in undergraduates' academic use of the internet. Active Learning in Higher Education, 9(11), 11-22.

Straub, D.; Keil, M. y Brenner, W. (1997). Testing the technology acceptance model across culture: A three country study. Information \& Management, 33(1), 1-11.

Straub, E. T. (2009). Understanding Technology Adoption: Theory and Future Directions for Informal Learning. Review of Educational Research, 79(2), 625- 649 .

Stryker, S. (1980). Symbolic interactionism: A social structural approach. Menlo Park: Benjamin \& Cummings.

Stryker, S. (1997). In the beginning there is society: Lessons from a sociological social psychology. En C. McGarty y S.A. Haslam (Eds.), The message of social psychology: Perspectives on mind society. Cambridge: Blackwell.

Stryker, S. y Burke, P.J. (2000). The past, present, and future of an identity theory. Social Psychology Quarterly, 63(4), 284-297.

Tapscott, D. (1998): Creciendo en un entorno digital. Bogotá: McGraw Hill.

Thinyane, H. (2010). Are digital natives a world-wide phenomenon? An investigation into South African first year students' use and experience with technology. Computers \& Education, 55(1), 406-414.

Turkle, S. (1984) The second self: Computers and the human spirit. New York: Simon and Schuster. 